\title{
Vom rechten Maß und seinen Folgen
}

Nicht erst seit die Digitalisierung und die Mobilitätswende über uns hereinbrechen, befinden sich große Teile unserer Wirtschaft im Wandel. Erfolgreiche Unternehmen passen sich veränderten Rahmenbedingungen an dieses Prinzip ist so alt wie der Handel selbst. Besonders erfolgreich waren dabei in den vergangenen Jahrzehnten die Zulieferer. Dass wir uns heute an komplexen Hightechprodukten erfreuen können, die selbst in ihren kleinsten Bestandteilen - man denke an Dichtungen oder Verbindungselemente - Ergebnisse jahrelanger Forschung widerspiegeln, ist insbesondere auch den zuliefernden Unternehmen zu verdanken. Es ist kein Zufall, dass Zulieferer beispielsweise in der deutschen Automobilindustrie 70 \% oder in der weltweiten Schienenfahrzeugindustrie 50 \% der Wertschöpfung erwirtschaften. Die Bedeutung dieser Hidden Champions für unseren Arbeitsmarkt ist dabei bekannt.

Kürzlich hörte ich einen Vortrag zur Zukunft des Leichtbaus. Der Referent forderte, eine neue Maßeinheit für den Leichtbau zu etablieren: das Funktionsgewicht. Oder wissenschaftlich korrekt: Masse pro Funktion. Das ist sinnvoll, denn bisher bewerten wir Leichtbaulösungen zumeist nach der Gewichtseinsparung gegenüber einem Referenzbauteil, etwa durch die Verwendung leichterer Werkstoffe. Doch wie bewer- tet man eine Struktur, die aus konventionellen Werkstoffen gebaut ist, aber die Funktion mehrerer Bauteile oder Systeme in einem einzigen vereint?

Wenn die Antwort das Funktionsgewicht ist, schließt sich eine weitere Frage an: Wer hat die Kompetenz für systemübergreifenden Leichtbau? Wer kann Einzelsysteme unterschiedlicher Funktionen zu einem einzigen zusammenführen? In erster Linie der OEM. Dem Zulieferer mit seinen herausragenden Einzelkomponenten fehlt das Systemverständnis, da er in die Entwicklung eines Autos oder Schienenfahrzeugs nicht hinreichend eingebunden ist. Dreht sich mit der Verschiebung der Leichtbaukompetenz hin zum OEM das Rad unserer diversifizierten, von kleinen und mittleren Unternehmen geprägten Wirtschaft wieder zurück?

Ganz so schlimm wird es für die KMUs wohl nicht kommen. Und dennoch sollten wir diese Entwicklung nicht auf die leichte Schulter nehmen. Gefordert sind neue Prozesse, die systematisch gewichtsoptimierte Produkte ermöglichen - und zwar in unternehmensübergreifenden Projekten. Ein solcher Prozess muss einerseits das Spezialwissen jedes Partners schützen, andererseits muss er so transparent sein, dass jeder Zulieferer versteht, wie sich seine Entwicklung auf das Gesamtprodukt auswirkt.

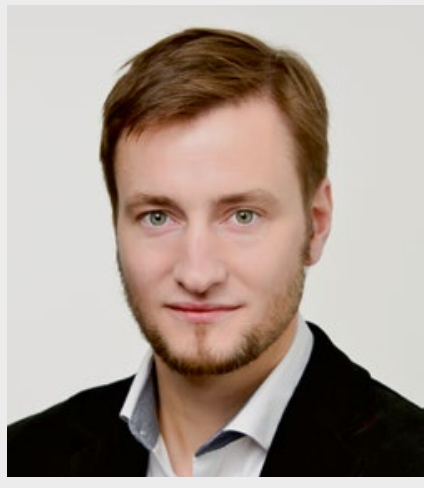

Thomas Siebel Verantwortlicher Redakteur, thomas.siebel@springer.com 